\title{
REMOCIÓN DE COLORANTES AZOICOS ROJO ALLURA (ROJO 40) MEDIANTE EL USO DE PERLAS DE QUITOSANO MAGNETIZADAS EN MEDIO ACUOSO
}

\author{
Fátima Amanda Santillán Espinozaa, Ily Marilú Maza Mejía*a
}

\begin{abstract}
RESUMEN
En este estudio se evaluó el uso de perlas de quitosano magnetizadas en la remoción de colorantes azoicos de la industria textil y alimentarias (Rojo Allura) tomando en cuenta factores como el $\mathrm{pH}$, concentración del colorante y cantidad de perlas magnetizadas. Para la obtención de las perlas se utilizó quitosano al $2 \%$, glutaraldehido y magnetita (previamente sintetizada mediante un proceso de hidrólisis oxidativa), posteriormente se caracterizaron las perlas y la magnetita mediante espectroscopía infrarroja y microscopía electrónica de barrido.
\end{abstract}

Palabras clave: remoción, colorantes, quitosano, magnetita.

\section{REMOVAL OF AZO DYES ALLURA RED (RED 40) BY USING MAGNETIC CHITOSAN BEADS IN AQUEOUS MEDIUM}

\begin{abstract}
In this study it was evaluated the use of magnetic chitosan beads in removal of textile and food azo dyes (Allura Red) taking into account some factors such as $\mathrm{pH}$, the concentration of the dye and the mass of beads. To obtain the beads it was used chitosan $2 \%$, glutaraldehyde and magnetite (previously synthetized by hydrolysis oxidative), after that the beads and magnetite were characterized by infrared spectroscopy and scanning electron microscopy.
\end{abstract}

Key words: removal, dyes, chitosan, magnetite.

\section{INTRODUCCIÓN}

En los últimos tiempos la calidad del agua ha sido un factor determinante del bienestar humano. El uso del agua contaminada ha afectado a millones de ciudadanos ya que pese a los grandes problemas que causa, gran parte de ellos se ven obligados a usarla ya sea para beber o para otros fines.

\footnotetext{
$\mathrm{a}^{*}$ Facultad de Ciencias, Escuela de Química, Universidad Nacional de Ingeniería Av. Túpac Amaru 210, Lima 25, Perú, ilyuni2002@yahoo.com.
} 
Aunque todavía hay epidemias de enfermedades bacterianas y virales causadas por agentes infecciosos transportados en el agua, la mayor preocupación en la actualidad es la presencia potencial de contaminantes químicos, entre los cuales encontramos a productos químicos orgánicos e inorgánicos, y metales pesados, procedentes de los diferentes tipos de industrias. ${ }^{1}$

La industria textil es una de las más importantes en el Perú, sin embargo, es una de las industrias con mayor consumo de agua y las aguas generadas a partir de ella contienen una gran cantidad de contaminantes, entre ellos los colorantes; los cuales son altamente resistentes incluso a la degradación microbiana, y es por ello que el uso de los tratamientos convencionales para su eliminación no es efectivo. ${ }^{2}$

Se ha encontrado que los colorantes azoicos como el Rojo Allura (Rojo 40) son causantes de reacciones alérgicas y de las alteraciones en el índice de hiperactividad en los niños, por lo cual es importante la propuesta de una alternativa para lidiar con ellos. ${ }^{3}$

Diversos estudios muestran que el quitosano entrecruzado presenta una gran eficiencia para remover colorantes en medios acuosos ${ }^{4-6}$. En este trabajo se desarrolló perlas de quitosano entrecruzadas y magnetizadas para aumentar tanto el porcentaje de remoción de colorantes como la fácil recuperación y no dejar productos secundarios después del proceso como otros tratamientos e incluso se propone la reutilización del material adsorbente. En resumen, este material presenta las ventajas de ser reusable, económico, estable y no tóxico al ambiente.

\section{PARTE EXPERIMENTAL}

\section{Síntesis de magnetita ${ }^{7}$}

Se preparó una solución KOH y KNO3 7:1 (w/w), la cual se hizo gotear sobre la solución ferrosa de FeSO4.7H2O 0,51 M y se dejó mezclando durante 30-60 minutos bajo atmósfera inerte de nitrógeno y en un baño María a $90^{\circ} \mathrm{C}$; luego se dejó enfriar para ser centrifugado y lavar el precipitado obtenido, finalmente se secó a temperatura ambiente.

\section{Obtención de perlas de quitosano magnetizadas ${ }^{8}$ (PQM)}

Se utilizó magnetita dispersada en una solución de quitosano $2 \%$ y ácido acético $1 \%$ utilizando un ultrasonido durante 30 minutos, esta solución obtenida se hizo gotear en $\mathrm{NaOH} 2 \mathrm{M}$, obteniéndose así las perlas de quitosano magnetizadas, las cuales fueron filtradas y lavadas. Estas perlas se dispersaron en una solución de acetona-agua 2:1 y se añadió glutaraldehído al $25 \%$ para finalmente obtener las perlas de quitosano magnetizadas entrecruzadas, que se lavaron con acetona.

\section{Caracterización de magnetitas y perlas de quitosano magnetizadas ${ }^{9}$}

Se utilizaron las técnicas de espectroscopía infrarroja y microscopía electrónica de barrido (SEM). En el caso de la espectroscopía infrarroja se realizó mediante dos métodos: reflectancia total atenuada (ATR) y reflectancia total difusa (DRS), esta última para una adecuada caracterización de magnetita, trabajándose ambas en un rango de $4000-400 \mathrm{~cm}^{-1}$. Para 
la investigación de la morfología de la magnetita y perlas se aplicó una película conductora a la superficie de cada muestra y se operó con el equipo SEM Zeiss EVO MA 10.

\section{Aplicación de perlas de quitosano magnetizadas (PQM) a los colorantes ${ }^{10}$}

Para la determinación del $\mathrm{pH}$ óptimo se utilizaron soluciones de colorante de $40 \mathrm{mg} / \mathrm{L}$ en un rango de pH de 2 a 10; a las cuales se le agregó 30 mg de PQM y se dejó agitando por 24 h, después se midió la absorbancia inicial y final de cada sorción $(\lambda=505,5 \mathrm{~nm})$ mediante curva de calibración de 2,5-20 mg/L utilizando un espectrofotómetro UV-Vis UV1800 Shimadzu.

Se trabajó con soluciones del colorante a concentraciones de 20, 50, 100, 150, 300 y 600 $\mathrm{mg} / \mathrm{L}$ para determinar la concentración adecuada del colorante en la remoción con PQM, a estas soluciones se le agregó 30 mg de PQM y se dejó agitando 24 h.

Para determinar la cantidad adecuada de PQM la remoción se trabajó con una solución de colorante de $40 \mathrm{mg} / \mathrm{L}$ y se agregó 10, 20, 40 y $80 \mathrm{mg}$ de PQM y se dejó agitando 24 h.

\section{RESULTADOS Y DISCUSIÓN}

La magnetita, obtenida mediante un proceso de hidrólisis oxidativa, no pierde sus propiedades magnéticas al momento de mezclarse con el quitosano para la obtención de las perlas de quitosano magnetizadas (figura 1).
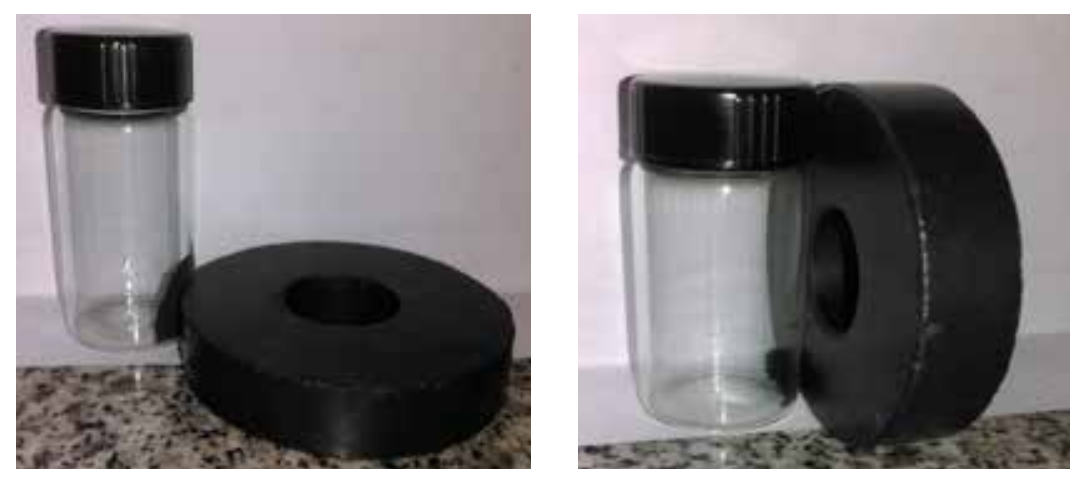

Figura 1. Magnetita sintetizada (izquierda), perlas de quitosano magnetizadas obtenidas (derecha). 


\section{Espectroscopía infrarroja}

Con la técnica de reflectancia difusa (DRS) se determinó las vibraciones de estiramiento y torsión de Fe-O (586 y $\left.416 \mathrm{~cm}^{-1}\right)$ correspondiente a la magnetita según la bibliografía ${ }^{9}$ como se puede apreciar en la figura 2.

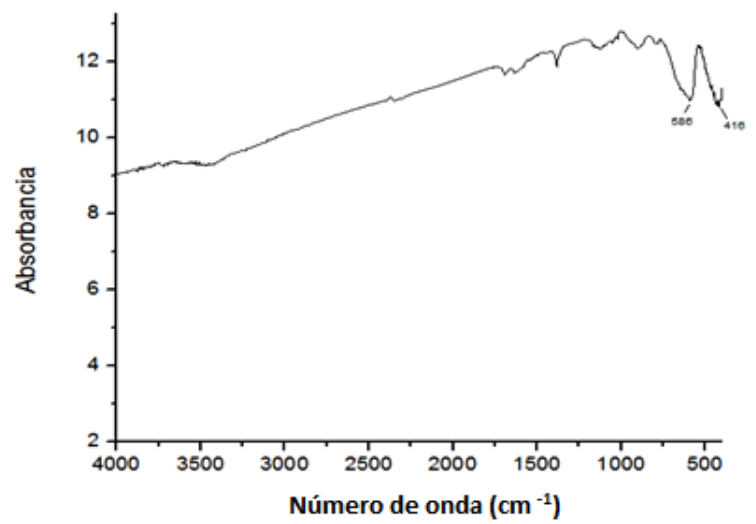

Figura 2. Espectro infrarrojo (DRS) de la magnetita sintetizada.

Mediante la técnica de reflectancia total atenuada (ATR) se obtuvieron los espectros que pueden apreciarse en la figura 3, donde aparecen los principales grupos funcionales del quitosano $^{11}$ (tabla 1) y la presencia de algunos de ellos de forma similar en las perlas de quitosano magnetizadas (tabla 2).

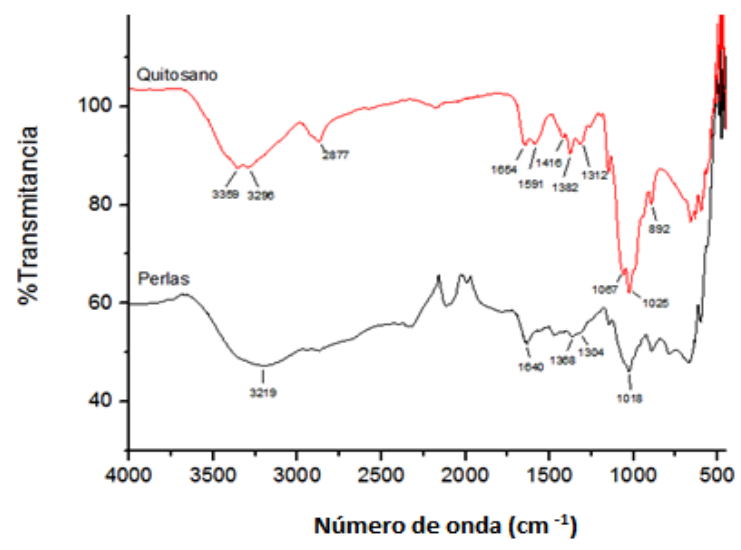

Figura 3. Espectros infrarrojos (ATR) de quitosano y perlas de quitosano magnetizadas (PQM). 
Tabla 1. Grupos funcionales característicos para el quitosano

\begin{tabular}{|l|c|c|c|}
\hline $\mathbf{N}^{\mathbf{0}}$ & Grupo Funcional & $\mathbf{c m}^{-\mathbf{1}}$ & Intensidad \\
\hline 1 & Grupo $-\mathrm{OH}$ & 3359 & $\mathrm{~V}$ \\
\hline 2 & Grupo $-\mathrm{NH}$ & 3296 & $\mathrm{~V}$ \\
\hline 3 & Grupo $-\mathrm{CH}$ & 2877 & $\mathrm{~F}$ \\
\hline 4 & Grupo Amida primaria & 1654 & $\mathrm{M}$ \\
\hline 5 & Grupo $-\mathrm{NH}_{2}$ & 1591 & $\mathrm{M}$ \\
\hline 6 & ${\mathrm{Grupo}-\mathrm{CH}_{2}}_{2}$ & 1416 & $\mathrm{M}$ \\
\hline 7 & $-\mathrm{CH}_{3}$ y C-CH & 1382 & $\mathrm{~F}$ \\
\hline 8 & Amida terciaria & 1312 & $\mathrm{~V}$ \\
\hline 9 & C-O-C & 1067 & $\mathrm{~F}$ \\
\hline 10 & Vibración del esqueleto & 1025 & $\mathrm{~V}$ \\
\hline 11 & $\begin{array}{c}\text { C-H de los grupos } \\
\text { monoméricos }\end{array}$ & 892 & $\mathrm{M}$ \\
\hline
\end{tabular}

F: Fuerte; M: Medio; V: Variable.

Tabla 2. Grupos funcionales característicos para las perlas de quitosano magnetizadas.

\begin{tabular}{|l|c|c|c|}
\hline $\mathbf{N}^{\mathbf{0}}$ & Grupo Funcional & $\mathbf{c m}^{-\mathbf{1}}$ & Intensidad \\
\hline 1 & Grupo $-\mathrm{OH}$ y $-\mathrm{NH}$ & 3219 & V \\
\hline 2 & Grupo $-\mathrm{NH}_{2}$ & 1640 & $\mathrm{M}$ \\
\hline 3 & $-\mathrm{CH}_{3}$ y C-CH & 1368 & $\mathrm{~F}$ \\
\hline 4 & Amida terciaria & 1304 & V \\
\hline 5 & C-O-C & 1018 & F \\
\hline
\end{tabular}

F: Fuerte, M: Medio, V: Variable.

\section{Microscopia electrónica de barrido (SEM)}

Mediante la técnica superficial de la microscopía electrónica de barrido (SEM) se obtuvo que la magnetita presentaba una distribución estrecha y se pudo notar la forma esférica de las perlas de quitosano magnetizadas además de determinar su tamaño, correspondiendo este a $1000 \mu \mathrm{m}$ (figura 4).

(a)

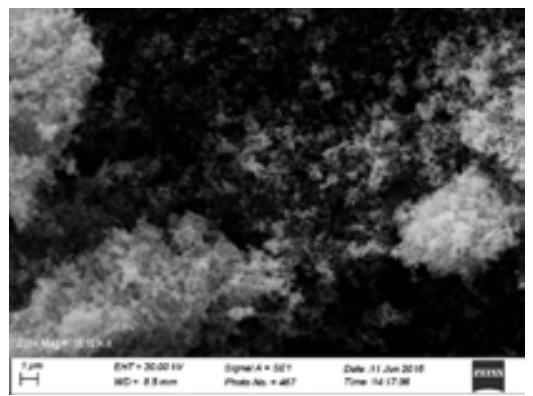

(b)

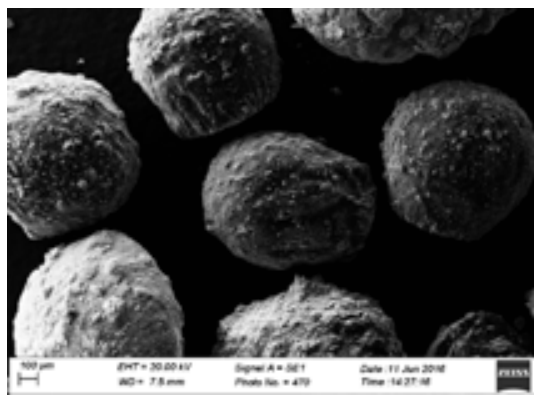

Figura 4. (a) Micrografías SEM de la magnetita, (b) de las perlas de quitosano magnetizadas. 


\section{Pruebas de sorción}

Para realizar las isotermas de sorción se debe establecer las condiciones óptimas del proceso, por lo cual se procede con la evaluación previa de cada uno de estos parámetros. Con respecto al pH óptimo, se encontró un mayor porcentaje de remoción a pHs ácidos comparado con los $\mathrm{pH}$ básicos, debido a que bajo estas condiciones los grupos aminos del quitosano se protonan y ocurre una interacción electrostática con los grupos de $\mathrm{SO}_{3-}$ del colorante como se muestra en la estructura del mismo permitiendo así la remoción (figura 5).
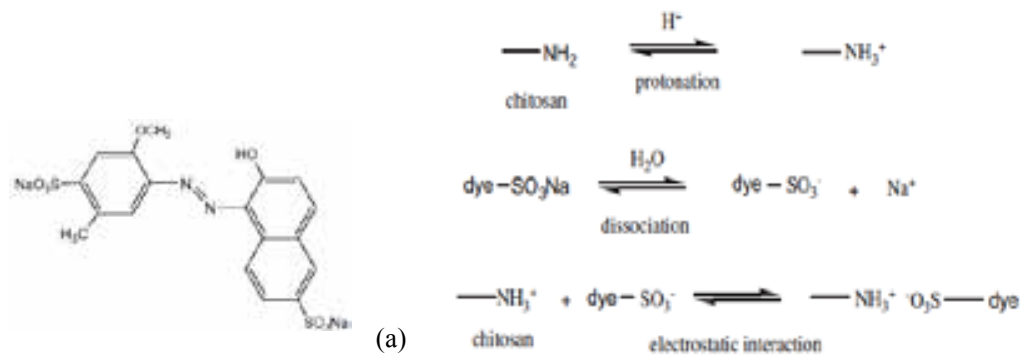

Figura 5. (a) Estructura del colorante Rojo Allura (Rojo 40)12 (b) Posible interacción de las PQM y el colorante13

Además, mediante una gráfica de \% remoción vs $\mathrm{pH}$ se obtuvo que el $\mathrm{pH}$ óptimo correspondiera a 2 ya que presentó el porcentaje más alto de remoción (figura 6).

(a)

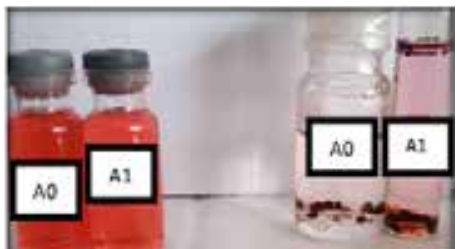

Figura 6. (a) Soluciones de Rojo 40 de $50 \mathrm{mg} / \mathrm{L}$ a pH 2 (Ao) y 3 (A1) antes y después de añadirles las PQM, (b) gráfica del \% remoción vs $\mathrm{pH}$.

A partir de las pruebas realizadas con soluciones de colorante a diferentes concentraciones, muestra una gran remoción a $20 \mathrm{mg} / \mathrm{L}$, pero realizando una gráfica de $\%$ de remoción frente a la cantidad de colorante se obtuvo que a $50 \mathrm{mg} / \mathrm{L}$ ocurría una saturación del material adsorbente, y es por ello que esta concentración se determina como la cantidad óptima del colorante para su remoción (figura 7). 
(a)

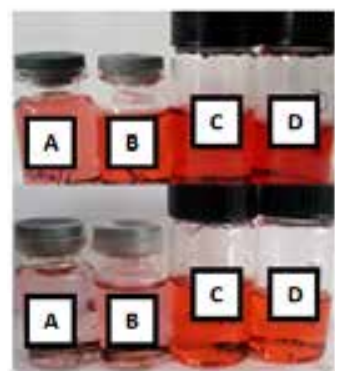

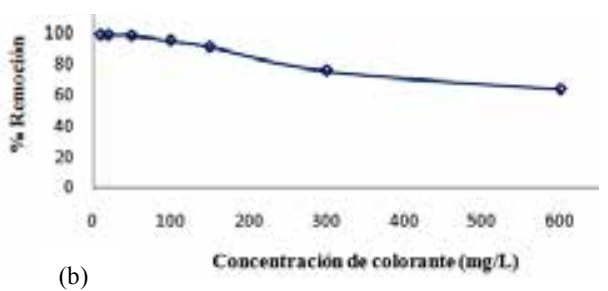

(b)

Figura 7. (a) Soluciones de Rojo $4020 \mathrm{mg} / \mathrm{L}$ (A), $50 \mathrm{mg} / \mathrm{L}$ (B), $100 \mathrm{mg} / \mathrm{L}$ (C) y $150 \mathrm{mg} / \mathrm{L}$ (D) antes y después de añadirles las PQM ,(b) gráfica de \% de remoción vs cantidad de colorante.

Mediante una gráfica del \% de remoción frente a la cantidad de perlas de quitosano magnetizadas agregada se obtuvo que a mayor cantidad de perlas añadidas ocurría un aumento de la remoción del colorante (teniendo en cuenta que la concentración es la misma para todas las soluciones), asimismo es notorio que agregando una mínima cantidad de perlas ocurría una gran remoción, porcentajes mayores al 90 \% (figura 8).

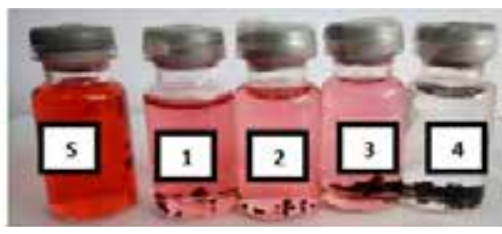

(a)

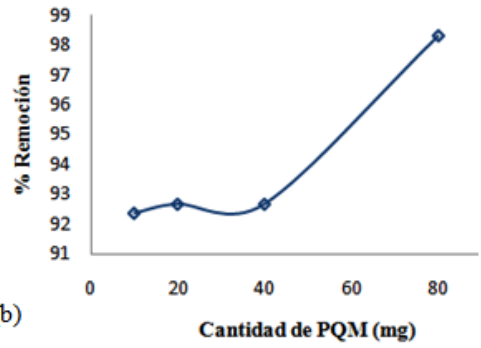

Figura 8. (a) Soluciones de Rojo $4050 \mathrm{mg} / \mathrm{L}$ (S), con $10 \mathrm{mg}$ (1); $20 \mathrm{mg}$ (2); $40 \mathrm{mg}$ (3) y 80 mg (4) de PQM, (b) gráfica de \% de remoción vs cantidad de PQM.

Luego de haber estudiado los factores como $\mathrm{pH}$, concentración del colorante y cantidad de perlas se obtuvo la isoterma de sorción (figura 9a), de la cual su gráfica linealizada bajo el modelo de Freundlich $(\log Q$ vs $\log C$ ) se obtuvo los valores de los parámetros k $(0,181)$ y $\mathrm{n}(1,034)$ que evidencian una quimisorción, pero como el valor de $\mathrm{n}$ es mayor a 1 indica que existe también una interacción física adsorbato-adsorbente (figura 9b). 

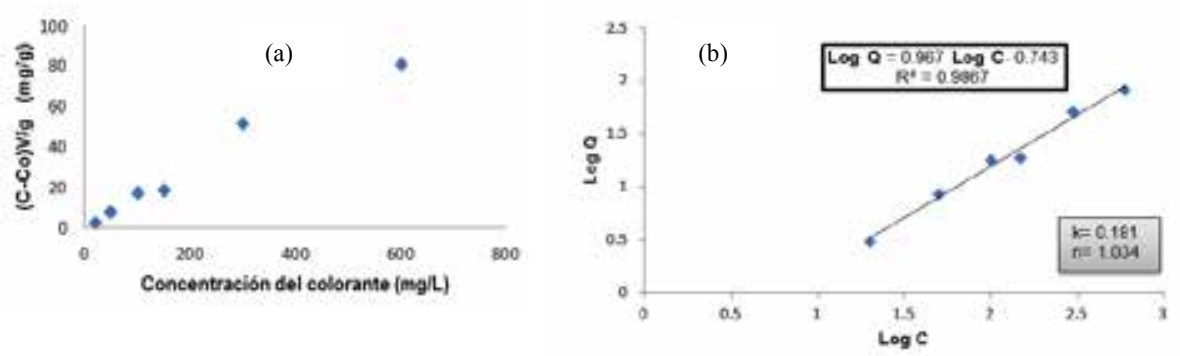

Figura 9. (a) Gráfica de la isoterma de sorción, (b) isoterma de sorción linealizada según el modelo de Freundlich.

\section{CONCLUSIONES}

Se obtuvieron las perlas de quitosano magnetizadas a partir de la magnetita preparada, que al ser entrecruzadas con glutaraldehído representan un buen aporte a la problemática ambiental debido a los altos porcentajes de remoción obtenidos (mayores al 90 \%) en la remoción del colorante azoico Rojo Allura (Rojo 40).

\section{AGRADECIMIENTO}

Agradezco al Laboratorio de Investigación de Análisis Instrumental y Medio Ambiente por brindarme sus instalaciones para la realización de los experimentos mostrados. Al Dr. José Díaz por las medidas realizadas en el microscopio electrónico de barrido.

\section{REFRERENCIAS BIBLIOGRÁFICAS}

1. Orozco S, Bandala E, Arancibia-Bulnes C, Serrano B, Suárez-Parra R, Hernández-Pérez I. Effect of iron salt on the color removal of water containing the azo-dye reactive blue 69 using photo-assisted Fe(II)/H2O2 and Fe(III)/H2O2 systems. J Photochem Photobiol A. 2008; 198 (2-3):144-149.

2. Barrios-Ziolo L, Gaviria-Restrepo L, Agudelo E, Cardona Gallo S. Tecnologías para la remoción de colorantes y pigmentos presentes en aguas residuales. DYNA. 2015; 82 (191):118-126.

3. Anjaneyulu Y, Sreedhara Chary N, Samuel Suman Raj D. Decolourization of Industrial Effluents - Available Methods and Emerging Technologies - A Review. Rev Environ Sci Biotechnol. 2005; 4 (4):245-273.

4. Chen A, Chen S. Biosorption of azo dyes from aqueous solution by glutaraldehydecrosslinked chitosans. J Hazard Mater. 2009; 172(2-3):1111-1121. 
5. Chiou M, Chuang G. Competitive adsorption of dye metanil yellow and RB15 in acid solutions on chemically cross-linked chitosan beads. Chemosphere. 2006; 62 (5):731740.

6. Du W, Xu Z, Han X, Xu Y, Miao Z. Preparation, characterization and adsorption properties of chitosan nanoparticles for eosin-Y as a model anionic dye. J Hazard Mater. 2008; 153 (1-2):152-156.

7. Picasso G, Vega J, Uzuriaga R, Ruiz G. preparación de nanopartículas de magnetita por los métodos sol-gel y precipitación: estudio de la composición química y estructura. Rev Soc Quim Peru. 2012; 78 (3):170-182.

8. Podzus P, Daraio M, Jacobo S. Chitosan magnetic microspheres for technological applications: Preparation and characterization. Phys Rev B.: Condens Matter. 2009; 404 (18):2710-2712.

9. Denkbaş E, Kiliçay E, Birlikseven C, Öztürk E. Magnetic chitosan microspheres: preparation and characterization. React Funct Polym. 2002; 50 (3):225-232.

10. Shen C, Shen Y, Wen Y, Wang H, Liu W. Fast and highly efficient removal of dyes under alkaline conditions using magnetic chitosan-Fe(III) hydrogel. Water Res. 2011; 45 (16):5200-5210.

11. Hernández H, Águila E, Flores O, Viveros E, Ramos E. Obtención y caracterización de quitosano a partir de exoesqueletos de camarón. Superficies y Vacío. 2009; 22 (3):5760.9

12. Villada Y, Hormaza A. Simultaneous analysis of the removal of brilliant blue and red 40 through spectrophotometric derivative. Ingeniería y Desarrollo. 2015; 33 (1):38-58. 10

13. Annadurai G, Lee D. Box-Behnken studies on dye removal from water using chitosan and activated carbon adsorbents. J Chin Inst Chem Engrs. 2000; 31 (6):609-615.11 